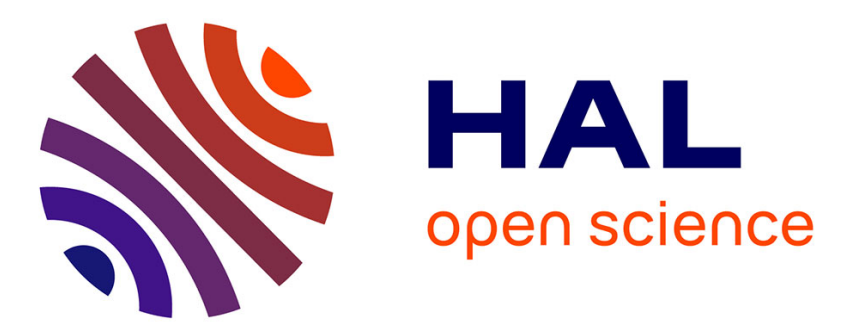

\title{
A Novel Iterative Receiver Design for CP-free Transmission under Frequency-Selective Channels
}

Roberto Bomfin, Marwa Chafii, Gerhard Fettweis

\section{To cite this version:}

Roberto Bomfin, Marwa Chafii, Gerhard Fettweis. A Novel Iterative Receiver Design for CP-free Transmission under Frequency-Selective Channels. IEEE Communications Letters, 2020, 24 (3), pp.525-529. 10.1109/LCOMM.2019.2959758 . hal-02538883

\section{HAL Id: hal-02538883 \\ https://hal.science/hal-02538883}

Submitted on 9 Apr 2020

HAL is a multi-disciplinary open access archive for the deposit and dissemination of scientific research documents, whether they are published or not. The documents may come from teaching and research institutions in France or abroad, or from public or private research centers.
L'archive ouverte pluridisciplinaire HAL, est destinée au dépôt et à la diffusion de documents scientifiques de niveau recherche, publiés ou non, émanant des établissements d'enseignement et de recherche français ou étrangers, des laboratoires publics ou privés. 


\title{
A Novel Iterative Receiver Design for CP-free Transmission under Frequency-Selective Channels
}

\author{
Roberto Bomfin, Marwa Chafii and Gerhard Fettweis, Fellow, IEEE
}

\begin{abstract}
In this paper, we develop a general iterative receiver based on the minimum mean squared error with parallel interference cancellation (MMSE-PIC) for cyclic prefix (CP)-free transmission. In particular, we consider that the receiver detects several blocks jointly, which allows backward block correction (BC). Additionally, we provide a structure with reduced complexity for Orthogonal Frequency Division Multiplexing (OFDM) and Orthogonal Chirp Division Multiplexing (OCDM) waveforms. As expected, the results show that our BC-based receiver improves the performance compared with a receiver without $\mathrm{BC}$. Moreover, we show that OCDM outperforms OFDM, and has practically no performance loss compared to CP-OFDM, which motivates the use of OCDM instead of CP-OFDM to decrease latency and increase spectral efficiency.
\end{abstract}

Index Terms-OCDM, OFDM, CP-free, MMSE-PIC.

\section{INTRODUCTION}

In wireless communication systems, CP-OFDM has been one of the most successful techniques to support high data rate transmission under frequency-selective channels (FSCs), due to its simplicity of one tap equalization in frequency-domain, which is only possible when a $\mathrm{CP}$ is added in time-domain before transmission. Clearly, introducing a $\mathrm{CP}$ implies extra overhead that reduces data rate and increases latency.

In order to deal with $\mathrm{CP}$ overhead, we are interested in investigating the transmission with no CP. Having no $\mathrm{CP}$ at the transmitter brings challenging negative effects such as interblock interference (IBI) and inter-carrier interference (ICI). Previous works in the literature approach these problems by employing the CP restoration procedure [1], [2]. In [1], the authors first partially restore the $\mathrm{CP}$ based on the samples from the next data block, and then perform the iterative $\mathrm{CP}$ restoration process of [3]. In [2], the CP restoration can be seen as a particular case of the one in [1], where CP is built completely from the next block's samples. In [4], another scheme has been proposed, however, this system relies on the channel state information at transmitter. In addition, another recent work has been reported in [5], which approaches the CP-free scheme with an artificial intelligence based solution.

In this work, we consider no channel state information at transmitter and we base our solution on the works done in

This work was supported by the European Union's Horizon 2020 under grant agreement no. 732174 (ORCA project), and by the Paris Seine Initiative (University of Cergy-Pontoise, CNRS, ENSEA, ETIS lab) through the ASIA Chair of Excellence Grant (PIA/ANR-16-IDEX-0008). The computations were performed at the Center for Information Services and High Performance Computing (ZIH) at Technische Universität Dresden.

Roberto Bomfin and Gerhard Fettweis are with the Vodafone Chair Mobile Communications Systems, Technische Universität Dresden, 01187 Dresden, Germany (e-mail: roberto.bomfin@ifn.et.tu-dresden.de; gerhard.fettweis@tudresden.de). Marwa Chafii is with ETIS, UMR 8051, Université Paris-Seine, Université Cergy-Pontoise, ENSEA, CNRS, France (email:marwa.chafii@ensea.fr)
[1], [2], in which we introduce an extra degree of freedom at the receiver that consists in allowing joint detection of several OFDM symbols. This new idea allows backward block correction when the systems of [1], [2] fail. Our solution consists of a general iterative receiver structure based on the MMSE-PIC [6] for block-based CP-free systems, which is applicable to any coded linear modulation scheme with soft-input soft-output (SISO) decoder. Additionally, we also evaluate the system beyond the OFDM waveform. For instance, in [7], the authors showed that the recently proposed CP-OCDM [8] is an optimal waveform for time and/or frequency selective channels if an iterative receiver that achieves the performance of perfect feedback equalizer is employed. This condition was achieved numerically in [9], where CP-OCDM shows $2 \mathrm{~dB}$ gain over CP-OFDM in terms of frame error rate (FER), proving that $\mathrm{CP}-\mathrm{OCDM}$ is indeed a very promising waveform for future communication systems. Thus, the findings of [7], [9] motivated us to compare OCDM with OFDM in this letter, where we provide MMSE-PIC implementation with reduced complexity for OFDM and OCDM.

A surprising outcome is that OCDM without $\mathrm{CP}$ has practically no performance loss in comparison to CP-OFDM, indicating that the CP-OFDM system can be replaced by our OCDM alternative with lower latency and higher spectral efficiency, with the penalty of increasing the complexity. However, we highlight that the CP-free and CP-OCDM systems already rely on an iterative structure with increased complexity. Another valuable and more general outcome of our experiments revealed that the system with partial CP restoration [1] is way more reliable than the one with full $\mathrm{CP}$ restoration [2], which is expected since [1] minimizes the signal-to-interference-noise ratio.

Notation: unless otherwise stated, column vectors are denoted with lowercase bold symbols $\mathbf{x}$ whose $i$-th element is denoted as $(\mathbf{x})_{i}$. The operation $[\mathbf{x}]_{n=a}^{b}=$ $\left((\mathbf{x})_{a},(\mathbf{x})_{a+1}, \cdots,(\mathbf{x})_{b}\right)^{\mathrm{T}}$ returns a subvector of $\mathbf{x}$, where $b>a .(\mathbf{x})^{-1}$ denotes element-wise inversion of the elements of $\mathbf{x}$. Matrices are written as uppercase bold symbols $\mathbf{X}$ whose element in $i$-th column and $j$-th row is $(\mathbf{X})_{i, j}$. $\operatorname{diag}(\mathbf{X})$ returns a column vector with elements being the diagonal of the matrix $\mathbf{X}$, and the row vector $(\mathbf{X})_{j, *}$ represents the $j$-th row of the matrix $\mathbf{X}$. The matrices $\mathbf{F}$ and $\mathbf{I}$ stand for normalized Fourier and identity matrices, respectively, whose sizes are implicitly given by the context. The column vectors $\mathbf{1}$ and $\mathbf{0}$ are the vectors of ones and zeros, respectively, with implicit sizes. $*, \circledast$ and $\circ$ denote the convolution, cyclic convolution and element-wise multiplication, respectively. 


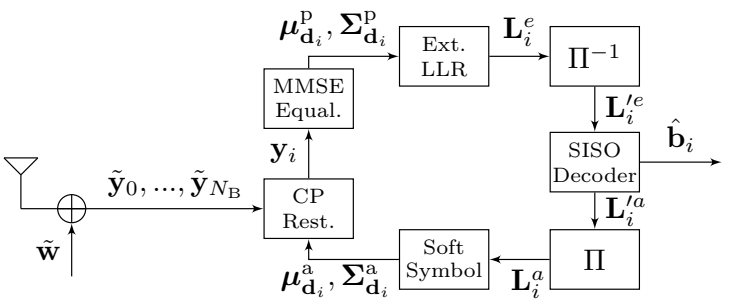

Fig. 1. Block diagram of iterative receiver.

\section{SYSTEM MODEL}

\section{A. Transmitter}

Consider a bit-interleaved coded modulation transmission. We assume the transmission of $N_{\mathrm{B}}$ blocks, where a vector of information bits transmitted in the $i$-th block is generated as $\mathbf{b}_{i}$. Subsequently, these bits are encoded and interleaved, generating the coded bit stream $\mathbf{c}_{i} \in\{0,1\}^{N_{\text {bits }}}$. In the sequence, the coded bits are mapped onto a quadrature amplitude modulation (QAM) constellation set $\mathcal{S}$ with cardinality $|\mathcal{S}|=J$, generating the data vector $\mathbf{d}_{i} \in \mathcal{S}^{N}$, where $N=N_{\text {bits }} / \log _{2} J$ is the amount of samples of a data block, $\mathbb{E}\left(\mathbf{d}_{i} \mathbf{d}_{i}^{\mathrm{H}}\right)=\mathbf{I}$ for all $i$ and $\mathbb{E}\left(\mathbf{d}_{i} \mathbf{d}_{j}^{\mathrm{H}}\right)=\mathbf{0}$ for all $i \neq j$. In general, the data symbols are linearly modulated as $\mathbf{x}_{i}=\mathbf{A}^{\prime} \mathbf{d}_{i}$, where $\mathbf{A}^{\prime} \in \mathbb{C}^{N \times N}$ is the transmission matrix in time domain. Finally, the transmitted signal is defined as a concatenation of $N_{\mathrm{B}}$ blocks with length $N, \mathbf{x}=\left(\mathbf{x}_{0}^{\mathrm{T}}, \mathbf{x}_{1}^{\mathrm{T}}, \cdots, \mathbf{x}_{N_{\mathrm{B}}-1}^{\mathrm{T}}\right)^{\mathrm{T}}$. In this paper, we consider the case where there is no $\mathrm{CP}$ insertion at the transmitter. Therefore, under the simplifying assumption of a perfect synchronization, the discrete-time received signal is given by

$$
\tilde{\mathbf{y}}=\mathbf{h} * \mathbf{x}+\mathbf{w}
$$

where $\mathbf{h}=\left(h_{0}, h_{1}, \cdots, h_{L_{\mathrm{ch}}-1}\right)^{\mathrm{T}}$ is the channel impulse response with length $L_{\mathrm{ch}}$, that is assumed to be known only at the receiver and to be invariant over the entire transmission time. $\mathbf{w} \sim \mathcal{C N}\left(0, \mathbf{I} \sigma^{2}\right)$ is the additive white Gaussian noise with variance $\sigma^{2}$.

\section{B. Receiver}

For simplicity, the following description assumes that the receiver waits for all $N_{\mathrm{B}}$ blocks to start detecting the data, whose structure is depicted in Fig. 1. However, in Section IV, we explain that the receiver only waits for the next data block where there is a detection error, which avoids an unnecessary latency increase and allows backward block correction. In other words, a block that is detected wrongly under a blockby-block detection can be correctly decoded by our proposed joint detection scheme. Moreover, this receiver can be seen as a generalization of the receiver proposed for the $\mathrm{CP}$-free transmission by [1] in two aspects. First, we allow backward block correction by jointly detecting two or more blocks. Secondly, our model is generalized to any linear modulation matrix, where we provide alternatives with reduced complexity for OFDM and OCDM modulations.

1) CP Restoration: First, we define the subvector based on the received signal (1) as $\tilde{\mathbf{y}}_{i}=[\tilde{\mathbf{y}}]_{n=i N}^{N(i+1)-1}$ for $0 \leq i \leq$ $N_{\mathrm{B}}-1$, and $\tilde{\mathbf{y}}_{i}=\left(\left([\tilde{\mathbf{y}}]_{n=i N}^{N i+L_{\mathrm{ch}}-1}\right)^{\mathrm{T}}, \mathbf{0}^{\mathrm{T}}\right)^{\mathrm{T}}$ for $i=N_{\mathrm{B}}$. In short, $\tilde{\mathbf{y}}_{i} \in \mathbb{C}^{N}$ is simply the arrangement of $N$ contiguous samples of $\tilde{\mathbf{y}}$ starting from $i N$-th sample. Notice that a vector of zeros is appended for $i=N_{\mathrm{B}}$ in order to guarantee a vector of length $N$. For convenience, we can write $\tilde{\mathbf{y}}_{i}$ as

$$
\tilde{\mathbf{y}}_{i}=\mathbf{H x}_{i}+\mathbf{H}^{\mathrm{p}} \mathbf{x}_{i-1}+\mathbf{w}_{i}
$$

where $\mathbf{H}^{\mathrm{p}} \in \mathbb{C}^{N \times N}$ shown in equation (3) is the interference matrix with respect to the previous $(i-1)$ block and the channel convolution matrix $\mathbf{H} \in \mathbb{C}^{N \times N}$ is defined as $\mathbf{H}=$ $\tilde{\mathbf{H}}-\mathbf{H}^{\mathrm{p}}$, where $\tilde{\mathbf{H}} \in \mathbb{C}^{N \times N}$ is the circulant channel matrix based on $\mathbf{h}$. Observe that the matrix $\mathbf{H}^{\mathrm{p}}$ can be seen as the missing part of the channel matrix $\tilde{\mathbf{H}}$ due to no $\mathrm{CP}$ insertion at transmitter. In addition, we define $\mathbf{x}_{-1}=\mathbf{x}_{N_{\mathrm{B}}}=\mathbf{0}$. Finally, $\tilde{\mathbf{w}}_{i} \sim \mathcal{C N}\left(0, \mathbf{I} \sigma^{2}\right)$ is the noise term.

$$
\mathbf{H}^{\mathrm{p}}=\left[\begin{array}{ccccc}
\cdots & 0 & h_{L_{\mathrm{ch}}-1} & \cdots & h_{1} \\
& & 0 & \ddots & \vdots \\
& & & & h_{L_{\mathrm{ch}}-1} \\
& & & & 0 \\
& & & & \vdots
\end{array}\right]
$$

Then, the CP restoration process consists of combining the samples of $\tilde{\mathbf{y}}_{i}$ and $\tilde{\mathbf{y}}_{i+1}$ linearly as

$$
\begin{aligned}
& \mathbf{y}_{i} \stackrel{(a)}{=} \tilde{\mathbf{y}}_{i}+\mathbf{R}_{i} \tilde{\mathbf{y}}_{i+1} \\
& \stackrel{(b)}{=} \mathbf{H} \mathbf{x}_{i}+\mathbf{H}^{\mathrm{p}} \mathbf{x}_{i-1}+\mathbf{w}_{i}+\mathbf{R}_{i} \mathbf{H} \mathbf{x}_{i+1}+\mathbf{R}_{i} \mathbf{H}^{\mathrm{p}} \mathbf{x}_{i}+\mathbf{R}_{i} \mathbf{w}_{i+1} \\
& \stackrel{(c)}{=}\left(\mathbf{H}+\mathbf{H}^{\mathrm{p}}\right) \mathbf{x}_{i}+ \\
& \left(\mathbf{R}_{i}-\mathbf{I}\right) \mathbf{H}^{\mathrm{p}} \mathbf{x}_{i}+\mathbf{H}^{\mathrm{p}} \mathbf{x}_{i-1}+\mathbf{R}_{i} \mathbf{H} \mathbf{x}_{i+1}+\mathbf{w}_{i}+\mathbf{R}_{i} \mathbf{w}_{i+1},
\end{aligned}
$$

where $\mathbf{R}_{i} \in \mathbb{R}_{\geq 0}^{N \times N}$ is the diagonal restoration matrix, whose elements were found with the help of the Appendix as

$$
\begin{aligned}
& (\mathbf{R})_{n, n}= \\
& \frac{\left(\mathbf{H}^{\mathrm{p}}\right)_{n, *} \mathbf{A} \boldsymbol{\Sigma}_{i}^{\mathrm{a}} \mathbf{A}^{\mathrm{H}}\left(\mathbf{H}^{\mathrm{p}}\right)_{n, *}^{\mathrm{H}}}{\left(\mathbf{H}^{\mathrm{p}}\right)_{n, *} \mathbf{A} \boldsymbol{\Sigma}_{i}^{\mathrm{a}} \mathbf{A}^{\mathrm{H}}\left(\mathbf{H}^{\mathrm{p}}\right)_{n, *}^{\mathrm{H}}+(\mathbf{H})_{n, *} \mathbf{A} \boldsymbol{\Sigma}_{i+1}^{\mathrm{a}} \mathbf{A}^{\mathrm{H}}(\mathbf{H})_{n, *}^{\mathrm{H}}+\sigma^{2}}
\end{aligned}
$$

for $0,1, \cdots N-1$, being $\Sigma_{i}^{a}$ the a-priori covariance matrix of symbols transmitted in the $i$-th block, which is described in Subsection II-B5. The equality $(a)$ in equation (4) takes the samples of the $(i+1)$-th block and add to the $i$-th block with the weights defined by the diagonal of $\mathbf{R}_{i}$. The second line (b) combines equations (2) and (4) (a). Further, the part $(c)$ separates $\mathbf{y}_{i}$ into two parts. The top line of $(c)$ represents the transmit signal affected by the convolutional channel matrix, $\tilde{\mathbf{H}}=\mathbf{H}+\mathbf{H}^{\mathrm{p}}$. And the bottom line represents the interference plus noise.

2) MMSE Equalizer: For convenience, we consider frequency domain processing, thus we apply fast Fourier transform (FFT) to the signal in (4) as

$$
\mathbf{Y}_{i}=\mathbf{F y}_{i}=\Lambda \mathbf{A d} \mathbf{d}_{i}+\mathbf{W}_{i},
$$

where $\boldsymbol{\Lambda}=\mathbf{F} \tilde{\mathbf{H}} \mathbf{F}^{\mathrm{H}}$ is a diagonal matrix whose elements are the channel response in frequency domain. $\mathbf{A}=\mathbf{F A}^{\prime}$ is the transmission matrix in frequency domain. And the noise plus interference term obtained from the bottom line of equation (4) $(c)$ is

$$
\mathbf{W}_{i}=\mathbf{F}\left(\left(\mathbf{R}_{i}-\mathbf{I}\right) \mathbf{H}^{\mathrm{p}} \mathbf{x}_{i}+\mathbf{H}^{\mathrm{p}} \mathbf{x}_{i-1}+\mathbf{R}_{i} \mathbf{H} \mathbf{x}_{i+1}+\mathbf{w}_{i}+\mathbf{R}_{i} \mathbf{w}_{i+1}\right) .
$$


We consider the component-wise conditionally unbiased (CWCU) MMSE-PIC [6]. Essentially, it computes the mean and error variance of $\mathbf{d}_{i}$, which are respectively given by

$$
\begin{aligned}
\boldsymbol{\mu}_{\mathbf{d}_{i}}^{\mathrm{p}}= & \frac{\mathbf{1}}{\mathbf{A}^{\mathrm{H}} \boldsymbol{\Lambda}^{\mathrm{H}}\left(\boldsymbol{\Lambda} \mathbf{A} \Sigma_{\mathbf{d}_{i}}^{\mathrm{a}} \mathbf{A}^{\mathrm{H}} \boldsymbol{\Lambda}^{\mathrm{H}}+\mathbf{R}_{\mathbf{W}_{i}}\right)^{-1} \boldsymbol{\Lambda} \mathbf{A}} \\
& \circ\left(\frac{\mathbf{A}^{\mathrm{H}} \boldsymbol{\Lambda}^{\mathrm{H}}\left(\mathbf{Y}_{i}-\boldsymbol{\Lambda} \mathbf{A} \boldsymbol{\mu}_{\mathbf{d}_{i}}^{\mathrm{a}}-\mathbb{E}\left(\mathbf{W}_{i}\right)\right)}{\boldsymbol{\Lambda} \mathbf{A} \boldsymbol{\Sigma}_{\mathbf{d}_{i}}^{\mathrm{a}} \mathbf{A}^{\mathrm{H}} \boldsymbol{\Lambda}^{\mathrm{H}}+\mathbf{R}_{\mathbf{W}_{i}}}\right)+\boldsymbol{\mu}_{\mathbf{d}_{i}}^{\mathrm{a}}
\end{aligned}
$$

and $^{1}$

$$
\begin{aligned}
\boldsymbol{\Sigma}_{\mathbf{d}_{i}}^{\mathrm{p}}= & \frac{\mathbf{1}}{\operatorname{diag}\left(\mathbf{A}^{\mathrm{H}} \boldsymbol{\Lambda}^{\mathrm{H}}\left(\boldsymbol{\Lambda} \mathbf{A} \boldsymbol{\Sigma}_{\mathbf{d}_{i}}^{\mathrm{a}} \mathbf{A}^{\mathrm{H}} \boldsymbol{\Lambda}^{\mathrm{H}}+\mathbf{R}_{\mathbf{W}_{i}}\right)^{-1} \boldsymbol{\Lambda} \mathbf{A}\right)} \\
& -\operatorname{diag}\left(\boldsymbol{\Sigma}_{\mathbf{d}_{i}}^{\mathrm{a}}\right),
\end{aligned}
$$

where $\boldsymbol{\mu}_{\mathbf{d}_{i}}^{\mathrm{a}}$ and $\boldsymbol{\Sigma}_{\mathbf{d}_{i}}^{\mathrm{a}}$ are the a-priori mean and covariance matrix of the $i$-th data vector, which are obtained from equations (11) and (12), respectively. Additionally, $\mathbb{E}\left(\mathbf{W}_{i}\right)=\mathbf{F}\left(\left(\mathbf{R}_{i}-\right.\right.$ I) $\left.\mathbf{H}^{\mathrm{p}} \mathbf{A}^{\prime} \boldsymbol{\mu}_{i}^{\mathrm{a}}+\mathbf{H}^{\mathrm{p}} \mathbf{A}^{\prime} \boldsymbol{\mu}_{i-1}^{\mathrm{a}}+\mathbf{R}_{i} \mathbf{H} \mathbf{A}^{\prime} \boldsymbol{\mu}_{i+1}^{\mathrm{a}}\right)$ is the mean of the a-priori interference term. $\mathbf{R}_{\mathbf{W}_{i}}=\mathbb{E}\left(\left(\mathbf{W}_{i}-\mathbb{E}\left(\mathbf{W}_{i}\right)\right)\left(\mathbf{W}_{i}-\right.\right.$ $\left.\left.\mathbb{E}\left(\mathbf{W}_{i}\right)\right)^{\mathrm{H}}\right)$ is the covariance matrix of the equivalent noise term, which can be expressed as ${ }^{2}$

$$
\begin{aligned}
\mathbf{R}_{\mathbf{W}_{i}}= & \mathbf{F}\left(\mathbf{R}_{i}-\mathbf{I}\right) \mathbf{H}^{\mathrm{p}} \mathbf{A}^{\prime} \Sigma_{\mathbf{d}_{i}}^{\mathrm{a}} \mathbf{A}^{\prime \mathrm{H}} \mathbf{H}^{\mathrm{pH}}\left(\mathbf{R}_{i}-\mathbf{I}\right)^{\mathrm{H}} \mathbf{F}^{\mathrm{H}}+ \\
& \mathbf{F} \mathbf{H}^{\mathrm{p}} \mathbf{A}^{\prime} \Sigma_{\mathbf{d}_{i-1}}^{\mathrm{a}} \mathbf{A}^{\prime \mathrm{H}} \mathbf{H}^{\mathrm{p} \mathrm{H}} \mathbf{F}^{\mathrm{H}}+\sigma^{2} \mathbf{F}\left(\mathbf{I}+\mathbf{R}_{i} \mathbf{R}_{i}^{\mathrm{H}}\right) \mathbf{F}^{\mathrm{H}} \\
& \mathbf{F R}_{i} \mathbf{H A}^{\prime} \boldsymbol{\Sigma}_{\mathbf{d}_{i+1}}^{\mathrm{a}} \mathbf{A}^{\prime \mathrm{H}} \mathbf{H}^{\mathrm{H}} \mathbf{R}_{i}^{\mathrm{H}} \mathbf{F}^{\mathrm{H}}
\end{aligned}
$$

3) Extrinsic LLR: Based on the outputs of the equalizer, $\boldsymbol{\mu}_{\mathbf{d}_{i}}^{\mathrm{p}}$ and $\boldsymbol{\Sigma}_{\mathbf{d}_{i}}^{\mathrm{p}}$, we compute the extrinsic log-likelihood ratios (LLRs) related to the $b$-th bit and $s$-th symbol of the $i$-th block using the low complexity method used in [6], [9]. As depicted in Fig. 1, the extrinsic LLR $\mathbf{L}_{i}^{e}$ serves as input for the SISO decoder after deinterleaving.

4) SISO Decoder: This step is responsible for calculating the a-priori LLR of the coded bits as $\mathbf{L}_{i}^{\prime a}$, which depends on the extrinsic LLRs $\mathbf{L}_{i}^{\prime e}$. In this work, we use a recursive systematic convolutional code with BCJR log-MAP decoder, as the performance of this coding scheme was reported to be successful [6], [9] for the MMSE-PIC. For the joint detection, when the blocks with indexes $(0, \cdots, i)$ are detected correctly or the maximum number of iterations is reached, the information bits are estimated as $\left(\hat{\mathbf{b}}_{0}, \cdots, \hat{\mathbf{b}}_{i}\right)$ by comparing the uncoded LLRs with zero. An example of joint detection is described in Section IV.

5) Soft Symbol: In this step, we compute the a-priori mean and variance of all $\mathbf{d}_{i}$ based on the a-priori LLRs, $\mathbf{L}_{i}^{a}$, which are provided by the SISO decoder. In short, we first calculate the bit probability based on the bit LLRs, and then compute the probability mass function of $s$-th symbol $\left(\mathbf{d}_{i}\right)_{s}$ as $\operatorname{Pr}\left\{\left(\mathbf{d}_{i}\right)_{s}=d\right\}$, for $d \in \mathcal{S}$. This standard procedure is found in [6], [9]. Then, the desired a-priori mean and variance of $\left(\mathbf{d}_{i}\right)_{s}$ are respectively computed as

$$
\left(\boldsymbol{\mu}_{\mathbf{d}_{i}}^{\mathrm{a}}\right)_{s}=\sum_{d \in \mathcal{S}} \operatorname{Pr}\left\{\left(\mathbf{d}_{i}\right)_{s}=d\right\} d,
$$

\footnotetext{
${ }^{1}$ For notational simplicity, the error variance in (9) is defined as a vector.

${ }^{2} \mathrm{We}$ omitted the intermediate steps due to lack of space.
}

$$
\text { and } \quad\left(\boldsymbol{\Sigma}_{\mathbf{d}_{i}}^{\mathrm{a}}\right)_{s, s}=\sum_{d \in \mathcal{S}} \operatorname{Pr}\left\{\left(\mathbf{d}_{i}\right)_{s}=d\right\}\left|d-\left(\boldsymbol{\mu}_{\mathbf{d}_{i}}^{\mathrm{a}}\right)_{s}\right|^{2},
$$

where $\boldsymbol{\mu}_{\mathbf{d}_{i}}^{\mathrm{a}} \in \mathbb{C}^{N}$ and $\boldsymbol{\Sigma}_{\mathbf{d}_{i}}^{\mathrm{a}} \in \mathbb{R}_{\geq 0}^{N}$. As in [6], the offdiagonal elements of $\boldsymbol{\Sigma}_{\mathbf{d}_{i}}^{\mathrm{a}}$ are set to zero. Notice that in the first iteration, there is no a-priori information available for the LLRs, thus $\left(\boldsymbol{\mu}_{\mathbf{d}_{i}}^{\mathrm{a}}\right)_{s}=0$ and $\left(\boldsymbol{\Sigma}_{\mathbf{d}_{i}}^{\mathrm{a}}\right)_{s, s}=1$ for all $(i, s)$.

\section{Relation to the schemes of [1] and [2]}

The method of [1] assumes no a-priori knowledge. Thus, we have $\boldsymbol{\Sigma}_{i}^{\mathrm{a}}=\mathbf{I} \forall i$. Consequently, the coefficients of (5) collapse into almost the same coefficients of [1, eq. (7)] with no $\mathrm{CP}^{3}$. Moreover, the receiver of [1] does not update the restoration matrix at each iteration as our general model, and the restoration of channel cyclicity is carried out by the iterative MMSE equalizer described in Subsection II-B2, with no knowledge of the $(i+1)$-th block.

For the scheme of [2], we set $\mathbf{R}_{i}=\mathbf{I} \forall i$, which we call full $\mathrm{CP}$ restoration, whereas the general case in (5) is a partial CP restoration. Differently from [1], the scheme of [2] does not rely on any iteration to reconstruct the channel the cyclicity, because the data already experiences a circular channel. Thus, the original system of [2] performs the MMSE only once. Notice that we are also able to assess the performance of the full $\mathrm{CP}$ restoration system with the backward correction, by keeping $\mathbf{R}_{i}=\mathbf{I} \forall i$ with knowledge of the $(i+1)$-th block.

\section{EQUALIZER WITH REDUCED COMPLEXITY}

Clearly, computation of (5), (8) and (9) are expensive due to the matrix inversion, which has in general a prohibitive complexity. Therefore, we provide a low complexity alternative for two different transmission matrices, namely, OFDM and OCDM.

The first task is to efficiently compute the covariance matrix $\mathbf{R}_{\mathbf{W}_{i}}$ in equation (10). One approach that is independent of the waveforms is to replace the covariance matrices $\Sigma_{\mathbf{d}_{i-1}}^{\text {a }}$ and $\boldsymbol{\Sigma}_{\mathbf{d}_{i+1}}^{\mathrm{a}}$ by the scalars $\sigma_{\mathbf{d}_{i-1}}^{2}=1 / N \sum_{n=1}^{N}\left(\boldsymbol{\Sigma}_{\mathbf{d}_{i-1}}^{\mathrm{a}}\right)_{n, n}$ and $\sigma_{\mathbf{d}_{i+1}}^{2}=1 / N \sum_{n=1}^{N}\left(\boldsymbol{\Sigma}_{\mathbf{d}_{i+1}}^{\mathrm{a}}\right)_{n, n}$. In short, this procedure neglects the individual a-priori variances by considering the averaged variance. This approximation has been shown to have no impact on the performance of CP-OCDM in [9], due to its spreading property. Yet another simplification is to utilize only the diagonal elements of the covariance matrix. In general, the off-diagonal elements of $\mathbf{R}_{\mathbf{W}_{i}}$ are small in comparison to the diagonal elements, therefore this simplification should not cause significant impact. Thus, given that $\mathbf{A}^{\prime} \mathbf{A}^{\prime H}=\mathbf{I}$ for the waveforms considered, we have

$$
\begin{aligned}
\left(\mathbf{R}_{\mathbf{W}_{i}}^{\prime}\right)_{n, n}= & \left(\sigma_{\mathbf{d}_{i-1}}^{2} \mathbf{F} \mathbf{H}^{\mathrm{p}} \mathbf{H}^{\mathrm{pH}} \mathbf{F}^{\mathrm{H}}+\sigma^{2} \mathbf{F}\left(\mathbf{I}+\mathbf{R}_{i} \mathbf{R}_{i}^{\mathrm{H}}\right) \mathbf{F}^{\mathrm{H}}\right. \\
& +\sigma_{\mathbf{d}_{i}}^{2} \mathbf{F}\left(\mathbf{R}_{i}-\mathbf{I}\right) \mathbf{H}^{\mathrm{p}} \mathbf{H}^{\mathrm{p} \mathrm{H}}\left(\mathbf{R}_{i}-\mathbf{I}\right)^{\mathrm{H}} \mathbf{F}^{\mathrm{H}} \\
& \left.+\sigma_{\mathbf{d}_{i+1}}^{2} \mathbf{F} \mathbf{R}_{i} \mathbf{H} \mathbf{H}^{\mathrm{H}} \mathbf{R}_{i}^{\mathrm{H}} \mathbf{F}^{\mathrm{H}}\right)_{n, n}
\end{aligned}
$$

for $n=0,1, \cdots, N-1$, and $\left(\mathbf{R}_{\mathbf{W}_{i}}^{\prime}\right)_{n, m}=0$ for $n \neq m$.

\footnotetext{
${ }^{3}$ Our solution is slightly different because we also take into consideration the noise term while it is neglected in [1].
} 
Additionally, we can also use the averaged a-priori variances to reduce the complexity of the restoration matrix in (5), which is given by

$$
\left(\mathbf{R}_{i}^{\prime}\right)_{n, n}=\frac{\sigma_{\mathbf{d}_{i}}^{2}\left(\mathbf{H}^{\mathrm{p}} \mathbf{H}^{\mathrm{p}^{\mathrm{H}}}\right)_{n, n}}{\sigma_{\mathbf{d}_{i}}^{2}\left(\mathbf{H}^{\mathrm{p}} \mathbf{H}^{\mathrm{p}^{\mathrm{H}}}\right)_{n, n}+\sigma_{\mathbf{d}_{i+1}}^{2}\left(\mathbf{H} \mathbf{H}^{\mathrm{H}}\right)_{n, n}+\sigma^{2}} .
$$

\section{A. OFDM}

In this case, we have $\mathbf{A}_{\text {OFDM }}^{\prime}=\mathbf{F}^{\mathrm{H}}$ and $\mathbf{A}_{\text {OFDM }}=\mathbf{I}$. Thus, we can rewrite (8) as

$$
\boldsymbol{\mu}_{\mathbf{d}_{i}}^{\mathrm{p}}=\frac{\boldsymbol{\Lambda}^{\mathrm{H}}\left(\boldsymbol{\Lambda} \boldsymbol{\Sigma}_{\mathbf{d}_{i}}^{\mathrm{a}} \boldsymbol{\Lambda}^{\mathrm{H}}+\mathbf{R}_{\mathbf{W}_{i}}^{\prime}\right)^{-1}\left(\mathbf{Y}_{i}-\mathbb{E}\left(\mathbf{W}_{i}\right)\right)}{\operatorname{diag}\left(\boldsymbol{\Lambda}^{\mathrm{H}}\left(\boldsymbol{\Lambda} \boldsymbol{\Sigma}_{\mathbf{d}_{i}}^{\mathrm{a}} \boldsymbol{\Lambda}^{\mathrm{H}}+\mathbf{R}_{\mathbf{W}_{i}}^{\prime}\right)^{-1} \boldsymbol{\Lambda}\right)}
$$

and

$$
\boldsymbol{\Sigma}_{\mathbf{d}_{i}}^{\mathrm{p}}=\frac{\mathbf{1}}{\operatorname{diag}\left(\boldsymbol{\Lambda}^{\mathrm{H}}\left(\boldsymbol{\Lambda} \boldsymbol{\Sigma}_{\mathbf{d}_{i}}^{\mathrm{a}} \boldsymbol{\Lambda}^{\mathrm{H}}+\mathbf{R}_{\mathbf{W}_{i}}^{\prime}\right)^{-1} \boldsymbol{\Lambda}\right)}-\operatorname{diag}\left(\boldsymbol{\Sigma}_{\mathbf{d}_{i}}^{\mathrm{a}}\right),
$$

where $\mathbb{E}\left(\mathbf{W}_{i}\right)=\mathbf{F}\left(\mathbf{R}_{i}-\mathbf{I}\right) \mathbf{H}^{\mathrm{p}} \mathbf{F}^{\mathrm{H}} \boldsymbol{\mu}_{i}^{\mathrm{a}}+\mathbf{F} \mathbf{H}^{\mathrm{p}} \mathbf{F}^{\mathrm{H}} \boldsymbol{\mu}_{i-1}^{\mathrm{a}}+$ $\mathbf{F R}_{i} \mathbf{H} \mathbf{F}^{\mathrm{H}} \boldsymbol{\mu}_{i+1}^{\mathrm{a}}$ Notice that $\mathbf{F H}^{\mathrm{p}}, \mathbf{F}\left(\mathbf{R}_{i}-\mathbf{I}\right) \mathbf{H}^{\mathrm{p}}$ and $\mathbf{F R}_{i} \mathbf{H}$ were computed in the calculation of $\mathbf{R}_{\mathbf{W}_{i}}^{\prime}$, and can be reused.

\section{B. $O C D M$}

For OCDM, the transmitter matrices in time and frequency are given by $\mathbf{A}_{\mathrm{OCDM}}^{\prime}=\mathbf{F}^{\mathrm{H}} \boldsymbol{\Gamma}^{\mathrm{H}} \mathbf{F}$ and $\mathbf{A}_{\mathrm{OCDM}}=\boldsymbol{\Gamma}^{\mathrm{H}} \mathbf{F}$, respectively, where $\boldsymbol{\Gamma} \in \mathbb{C}^{N}$ is a diagonal matrix whose elements are given by $\left[\exp \left(-j \pi n^{2} / N\right)\right]_{n=0}^{N-1}$ [8], [9]. Thus, we can write the equalizer similarly to [9] as

$$
\boldsymbol{\mu}_{\mathbf{d}_{i}}^{\mathrm{p}}=\boldsymbol{\mu}_{\mathbf{d}_{i}}^{\mathrm{a}}+\frac{\mathbf{F}^{\mathrm{H}} \boldsymbol{\Gamma} \boldsymbol{\Lambda}^{\mathrm{H}}\left(\mathbf{Y}_{i}-\boldsymbol{\Lambda} \boldsymbol{\Gamma}^{\mathrm{H}} \mathbf{F} \boldsymbol{\mu}_{\mathbf{d}_{i}}^{\mathrm{p}}-\mathbb{E}\left(\mathbf{W}_{i}\right)\right)}{\lambda_{\text {norm }}\left(\sigma_{\mathbf{d}_{i}}^{2} \boldsymbol{\Lambda} \boldsymbol{\Lambda}^{\mathrm{H}}+\mathbf{R}_{\mathbf{W}_{i}}^{\prime}\right)}
$$

and

$$
\Sigma_{\mathbf{d}_{i}}^{\mathrm{p}}=\frac{1}{\lambda_{\text {norm }}}-\sigma_{\mathbf{d}_{i}}^{2} \mathbf{1}
$$

where $\boldsymbol{\Sigma}_{\mathbf{d}_{i}}^{\mathrm{a}}$ has been replaced by $\sigma_{\mathbf{d}_{i}}^{2}=1 / N \sum_{n=1}^{N}\left(\boldsymbol{\Sigma}_{\mathbf{d}_{i}}^{\mathrm{a}}\right)_{n, n}$. It has been shown in [9] that this modification provokes no performance loss and reduces complexity considerably. In [9, eq. (10)], the authors showed that due to the definition of the FFT matrix $\mathbf{F}$, the normalization term $\lambda_{\text {norm }}$ is easily computed as $\lambda_{\text {norm }}=1 / N \operatorname{Tr}\left(\boldsymbol{\Lambda}^{\mathrm{H}}\left(\sigma_{\mathbf{d}_{i}}^{2} \boldsymbol{\Lambda} \boldsymbol{\Lambda}^{\mathrm{H}}+\mathbf{R}_{\mathbf{W}_{i}}^{\prime}\right) \boldsymbol{\Lambda}\right)$. Additionally, we have $\mathbb{E}\left(\mathbf{W}_{i}\right)=\mathbf{F}\left(\mathbf{R}_{i}-\mathbf{I}\right) \mathbf{H}^{\mathrm{p}} \mathbf{F}^{\mathrm{H}} \boldsymbol{\Gamma}^{\mathrm{H}} \mathbf{F} \boldsymbol{\mu}_{i}^{\mathrm{a}}+$ $\mathbf{F} \mathbf{H}^{\mathrm{p}} \mathbf{F}^{\mathrm{H}} \boldsymbol{\Gamma}^{\mathrm{H}} \mathbf{F} \boldsymbol{\mu}_{i-1}^{\mathrm{a}}+\mathbf{F} \mathbf{R}_{i} \mathbf{H F}^{\mathrm{H}} \boldsymbol{\Gamma}^{\mathrm{H}} \mathbf{F} \boldsymbol{\mu}_{i+1}^{\mathrm{a}}$.

We do not provide a detailed complexity analysis due to the lack of space. However, in general we can conclude that OCDM needs three times more FFTs than OFDM, since it needs two more FFTs to compute $\mathbb{E}\left(\mathbf{W}_{i}\right)$ and one more in equation (17). In addition, we highlight that the MMSE-PIC with joint detection described in this section requires more complexity than the schemes of [1] and [2], with partial and full $\mathrm{CP}$ restoration, respectively. However, as we explain in the following section, the extra computation can be requested only when a block is wrongly detected, i.e., when [1] or [2] fail, which is assessed by a cyclic redundancy check (CRC).

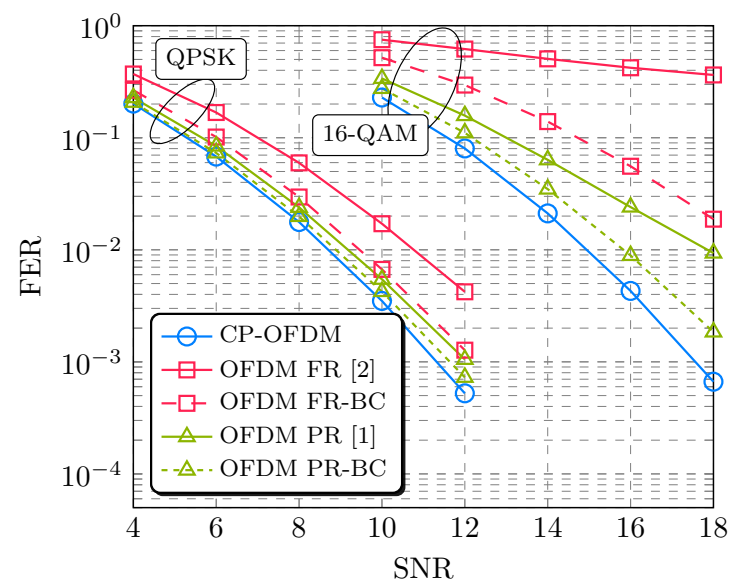

Fig. 2. FER of (CP)-OFDM for different receivers, namely partial (PR) and full (FR) CP restoration, with and without backward correction (BC).

\section{Numerical Evaluation AND Discussion}

For the simulation setup, we consider a fully allocated system with $N=64$ samples, $N_{\mathrm{B}}=4$ blocks and bandwidth (sampling rate) of $B=4.32 \mathrm{MHz}$. The channel is perfectly known at the receiver and invariant over the transmission of $N_{\mathrm{B}}$ blocks. Its impulse response is obtained from independent complex Gaussian random variables, whose powers and delays are defined by the extended vehicular-A (EVA) channel model [6]. The channel length is 12 samples and we consider $N_{\mathrm{CP}}=16$ samples for CP-OFDM/CP-OCDM. Additionally, we assume a bit-interleaved coded modulated system with $1 / 2$ code rate $\{133,171\}_{8}$ convolutional encoder, that interleaves and maps the bits onto either QPSK or 16QAM constellations, respectively, and where we perform a maximum number of iterations as 7 and 10, for QPSK and 16QAM, respectively.

First, we evaluate our scheme with the ones provided in [1] and [2], where our receiver brings the new feature of backward correction (BC) for both full and partial CP restoration. For the system with $\mathrm{BC}$ and full $\mathrm{CP}$ restoration, we always keep $\mathbf{R}_{i}=\mathbf{I} \forall i$. In addition, we perform the joint detection only when a block is not detected correctly. For example, we start by detecting the block $i=0$ with no knowledge about the data of block $i=1$. If the block $i=0$ is not detected correctly ${ }^{4}$, the receiver waits for the block $i=1$ and then performs joint detection of $i=\{0,1\}$. Otherwise, the receiver moves forward and starts detecting the block $i=1$ individually. In case the receiver still does not detect the block $i=0$ correctly, it waits for the block $i=2$ and then performs joint detection of $i=$ $\{0,1,2\}$. We assume that the receiver is capable of handling all $N_{\mathrm{B}}$ blocks jointly, in the worst case.

The outcomes are depicted in Fig. 2. The first observation to make is that the partial $\mathrm{CP}$ restoration (PR) [1] provides a very high performance improvement in relation to the full $\mathrm{CP}$ restoration (FP) [2], regardless of the backward correction (BC) technique. Thus, a major conclusion is that the interference power minimization proposed by [1] and generalized in this paper is crucial for the $\mathrm{CP}$-free transmission. We highlight

\footnotetext{
${ }^{4}$ The receiver performs the CRC in a block basis in order to check whether the block is correct or not.
} 


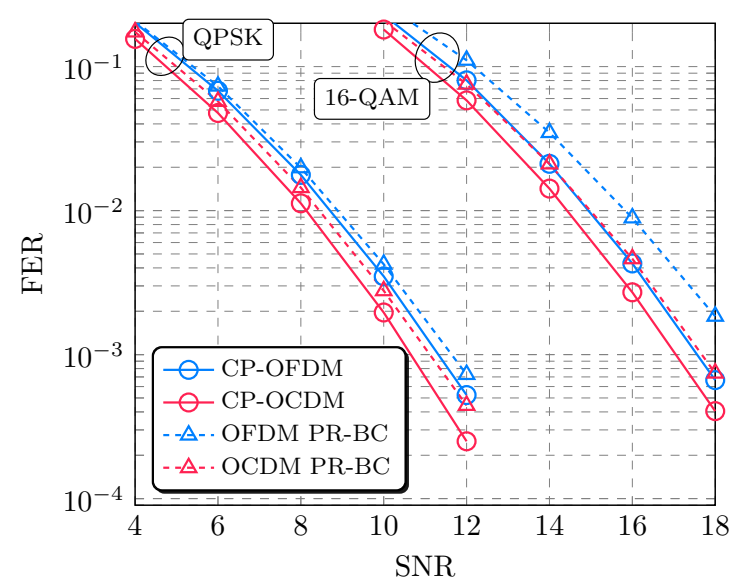

Fig. 3. FER of (CP)-OFDM and (CP)-OCDM for partial CP restoration with backward correction.

that [2] overestimated the system's performance due to the unrealistic assumption of correct detection of the $(i-1)$-th block, and no interference from $(i+1)$-th block. Regarding the $\mathrm{BC}$ scheme introduced in this paper, we observe that it improves the performance in relation to [1] and [2]. This performance gain is justified by the extra degree of freedom. For the partial CP restoration system, the performance improvement is more significant for the 16-QAM system, with a $2 \mathrm{~dB}$ gain for $\mathrm{FER}=10^{-2}$, while for the full $\mathrm{CP}$ restoration technique, the improvement is considerable for both QPSK and 16-QAM.

Fig. 3 compares (CP)-OFDM and (CP)-OCDM, where we selected to employ the receiver based on the partial $\mathrm{CP}$ restoration with $\mathrm{BC}$ due to its good performance. As expected from [9], the results reveal that (CP)-OCDM outperforms (CP)-OFDM in all cases. More importantly, the outcomes reveal that OCDM has practically no performance loss even in relation to CP-OFDM, meaning that the conventional CPOFDM modulation can be replaced by OCDM with no performance degradation, and with more spectral efficiency and reduced latency.

\section{CONClusion}

We have derived a general iterative receiver based on the MMSE-PIC for CP-free transmission under frequencyselective channels. Our contributions were twofold. First, we have introduced the receiver with backward correction (BC), where we can correct erroneous blocks by jointly detecting more than one block. Secondly, we have provided a general equalizer for any linear modulation matrix, with reduced complexity solution for OFDM and OCDM waveforms.

As expected, the results revealed that the $\mathrm{BC}$ technique improves the performance for OFDM in comparison to current models available in the literature. The gain is more evident with higher modulation order. Comparing OCDM with OFDM, we observed that OCDM shows better or practically equal performance to CP-OFDM, for QPSK and 16-QAM, respectively. In other words, the classical CP-OFDM modulation can be replaced by OCDM with no performance degradation, but with more spectral efficiency and reduced latency, which is significantly beneficial for low-latency and enhanced mobile broadband communications.

\section{APPENDIX}

Following the same idea of [1], our goal is to derive the coefficients of $\mathbf{R}_{n, n}$ such that the power of interference plus noise is minimized. From equation (4)(c), the $n$-th sample of the signal containing noise and interference is given by

$$
\begin{aligned}
\left(\tilde{\mathbf{w}}_{i}\right)_{n}= & \left(\left(\mathbf{R}_{i}\right)_{n, n}-1\right)\left(\mathbf{H}^{\mathrm{p}}\right)_{n, *} \mathbf{A} \mathbf{d}_{i}+\left(\mathbf{w}_{i}\right)_{n}+\left(\mathbf{R}_{i}\right)_{n, n}\left(\mathbf{w}_{i+1}\right)_{n} \\
& +\left(\mathbf{H}^{\mathrm{p}}\right)_{n, *} \mathbf{A} \mathbf{d}_{i-1}+\left(\mathbf{R}_{i}\right)_{n, n}(\mathbf{H})_{n, *} \mathbf{A} \mathbf{d}_{i+1},
\end{aligned}
$$

for $n=0,1, \cdots N-1$, and whose expected value is given by

$$
\begin{aligned}
\mathbb{E}\left(\left(\tilde{\mathbf{w}}_{i}\right)_{n}\right)= & \left(\left(\mathbf{R}_{i}\right)_{n, n}-1\right)\left(\mathbf{H}^{\mathrm{p}}\right)_{n, *} \mathbf{A} \boldsymbol{\mu}_{\mathbf{d}_{i}}^{\mathrm{a}} \\
& +\left(\mathbf{H}^{\mathrm{p}}\right)_{n, *} \mathbf{A} \boldsymbol{\mu}_{\mathbf{d}_{i-1}}^{\mathrm{a}}+\left(\mathbf{R}_{i}\right)_{n, n}(\mathbf{H})_{n, *} \mathbf{A} \boldsymbol{\mu}_{\mathbf{d}_{i+1}},
\end{aligned}
$$

where $\boldsymbol{\mu}_{\mathbf{d}_{i}}^{\mathrm{a}}$ is the a-priori mean of the data vector transmitted in the $i$-th block, and is defined in Subsection II-B5.

Combining equations (19) and (20), we calculate the variance of $\left(\tilde{\mathbf{w}}_{i}\right)_{n}$ as a function of $\left(\mathbf{R}_{i}\right)_{n, n}$

$$
\begin{aligned}
V\left(\left(\tilde{\mathbf{w}}_{i}\right)_{n}\right) & =\mathbb{E}\left(\left|\left(\tilde{\mathbf{w}}_{i}\right)_{n}-\mathbb{E}\left(\left(\tilde{\mathbf{w}}_{i}\right)_{n}\right)\right|^{2}\right) \\
& =\left(\mathbf{H}^{\mathrm{p}}\right)_{n, *} \mathbf{A} \boldsymbol{\Sigma}_{i-1}^{\mathrm{a}} \mathbf{A}^{\mathrm{H}}\left(\mathbf{H}^{\mathrm{p}}\right)_{n, *}^{\mathrm{H}}+\left(1+\left(\mathbf{R}_{i}\right)_{n, n}^{2}\right) \sigma^{2} \\
& +\left(\left(\mathbf{R}_{i}\right)_{n, n}-1\right)^{2}\left(\mathbf{H}^{\mathrm{p}}\right)_{n, *} \mathbf{A} \boldsymbol{\Sigma}_{i}^{\mathrm{a}} \mathbf{A}^{\mathrm{H}}\left(\mathbf{H}^{\mathrm{p}}\right)_{n, *}^{\mathrm{H}} \\
& +\left(\mathbf{R}_{i}\right)_{n, n}^{2}(\mathbf{H})_{n, *} \mathbf{A} \boldsymbol{\Sigma}_{i+1}^{\mathrm{a}} \mathbf{A}^{\mathrm{H}}(\mathbf{H})_{n, *}^{\mathrm{H}},
\end{aligned}
$$

in which $\Sigma_{i}^{a}$ is the a-priori covariance matrix of symbols transmitted in the $i$-th block, obtained in Subsection II-B5.

In the sequence, we compute the derivative of $V\left(\left(\tilde{\mathbf{w}}_{i}\right)_{n}\right)$ with respect to $\left(\mathbf{R}_{i}\right)_{n, n}$ as

$$
\begin{aligned}
& \frac{\partial V\left(\left(\tilde{\mathbf{w}}_{i}\right)_{n}\right)}{\partial\left(\mathbf{R}_{i}\right)_{n, n}}=2\left(\left(\mathbf{R}_{i}\right)_{n, n}-1\right)\left(\mathbf{H}^{\mathrm{p}}\right)_{n, *} \mathbf{A} \boldsymbol{\Sigma}_{i}^{\mathrm{a}} \mathbf{A}^{\mathrm{H}}\left(\mathbf{H}^{\mathrm{p}}\right)_{n, *}^{\mathrm{H}} \\
& \quad+2\left(\mathbf{R}_{i}\right)_{n, n} \sigma^{2}+2\left(\mathbf{R}_{i}\right)_{n, n}(\mathbf{H})_{n, *} \mathbf{A} \boldsymbol{\Sigma}_{i+1}^{\mathrm{a}} \mathbf{A}^{\mathrm{H}}(\mathbf{H})_{n, *}^{\mathrm{H}} \cdot
\end{aligned}
$$

Setting the derivative to zero, the value of $\left(\mathbf{R}_{i}\right)_{n, n}$ that minimizes (21) is found as (5).

\section{REFERENCES}

[1] Cheol-Jin Park and Gi-Hong Im, "Efficient Cyclic Prefix Reconstruction for Coded OFDM Systems," IEEE Commun. Lett., vol. 8, pp. 274-276, May 2004.

[2] X. Liu, H. Chen, S. Chen, and W. Meng, "Symbol Cyclic-Shift Equalization Algorithm-A CP-Free OFDM/OFDMA System Design," IEEE Trans. Veh. Technol., vol. 66, pp. 282-294, Jan 2017.

[3] Dukhyun Kim and G. L. Stuber, "Residual ISI Cancellation for OFDM with Applications to HDTV Broadcasting," IEEE J. Sel. Areas Commun., vol. 16, pp. 1590-1599, Oct 1998 .

[4] J. M. Hamamreh, Z. E. Ankarali, and H. Arslan, "CP-Less OFDM With Alignment Signals for Enhancing Spectral Efficiency, Reducing Latency, and Improving PHY Security of 5G Services," IEEE Access, vol. 6, pp. 63649-63663, 2018.

[5] J. Zhang, C. Wen, S. Jin, and G. Y. Li, "Artificial intelligence-aided receiver for a cp-free ofdm system: Design, simulation, and experimental test," IEEE Access, vol. 7, pp. 58901-58914, 2019.

[6] M. Matthé, D. Zhang, and G. Fettweis, "Low-Complexity Iterative MMSE-PIC Detection for MIMO-GFDM," IEEE Trans. Commun., vol. 66, pp. 1467-1480, April 2018.

[7] R. Bomfin, D. Zhang, M. Matthé, and G. Fettweis, "A Theoretical Framework for Optimizing Multicarrier Systems Under Time and/or Frequency-Selective Channels," IEEE Commun. Lett., vol. 22, pp. 2394 2397, Nov 2018.

[8] X. Ouyang and J. Zhao, "Orthogonal Chirp Division Multiplexing," IEEE Trans. Commun., vol. 64, pp. 3946-3957, Sept 2016.

[9] R. Bomfin, M. Chafii, and G. Fettweis, "Low-Complexity Iterative Receiver for Orthogonal Chirp Division Multiplexing," in IEEE WCNC workshops, (Marrakech, Morocco), Apr. 2019. 